\title{
Emergency Mesh Repair of Incarcerated Umbilical Hernias using Prosthetic Mesh: Outcomes of Early Surgical Complications And Recurrence
}

\author{
Alp Yildiz ${ }^{1 *}$, Mesut Yavaş ${ }^{2}$, Can Sahin ${ }^{2}$ and Aybala Yildiz ${ }^{1}$ \\ ${ }^{1}$ Department of General Surgery, Yenimahalle Training and Research Hospital, Turkey \\ ${ }^{2}$ Department of General Surgery, Gazi University School of Medicine, Turkey \\ *Corresponding Author: Alp Yildiz, Department of General Surgery, Yenimahalle Training and Research Hospital, Turkey.
}

Received: June 21, 2019; Published: July 10, 2019

DOI: $10.31080 /$ ASGIS.2019.02.0057

\begin{abstract}
The concept of repair of umbilical hernia in adults is not new, but the choice of appropriate surgical procedure is still a subject of debate. Despite umbilical hernia being common, there are no set surgical guidelines for its repair and there is no consensus on the best type of repair. Especially emergency repair of umbilical hernias still a matter of debate. In this study we present our outcomes of surgical complications and recurrence rates of emergency tension-free repair of 9 consecutive cases. There was no significant difference of recurrence rates and wound complications between literature and our series supporting tension-free mesh repair of incarcerated umbilical hernias as a safe and effective procedure but still more studies with larger patient series needed.
\end{abstract}

Keywords: Repair; Umbilical Hernias; Recurrence

\section{Introduction}

Umbilical hernias account for approximately $6 \%$ to $14 \%$ of all abdominal wall hernias in adults, and almost $90 \%$ of adult umbilical hernias are acquired. Despite umbilical hernia being common, there are no set surgical guidelines for its repair and there is no consensus on the best type of repair. Recurrence rates range from $1 \%$ to $43 \%$, but the literature offers little consensus on factors that affect recurrence and surgical complications [1]. In this study we review the complication and recurrence outcomes of our emergency herniorrhaphy patients with literature.

\section{Patients and Methods}

This was a clinical study done in the department of surgery in a tertiary care hospital. A total of 9 patients included the study. The study was approved by the Institutional Ethical Committee. All the patients who attended emergency department with incarcerated yet not strangulated umbilical hernia (UH) underwent emergency herniorrhaphy were enrolled in our study. Patients with abdominal malignancies, Patients with coagulopathy, severe cardiopulmonary disease, ascites and renal failure, Patients who had UH repair in combination with another major surgical operation such as laparoscopic cholecystectomy and inguinal hernia repair excluded from study. All patients operated by same surgeon. All cases were done under general anaesthesia. Antibiotic was prophylactically given before incision and two doses given postoperatively. Urinary bladder catheterization is done in all groups. an inferior para-Umbilica/transverse supraumbilical incision was made. The sac was dissected and freed up to its neck. Where it was opened, the con- tacts was reduced and the sac was ligated at its neck. Aponeurotic plain was cleared from subcutaneous tissue about $3-4 \mathrm{~cm}$ around the border of the defect. Dual-sided mesh (polymesh dual ${ }^{\circledR}$, Betatech Medical,Istanbul/Turkey) was cut according to the size of the defect extending $3 \mathrm{~cm}$ beyond the margins of the defect. The mesh covering the defect was placed over the anterior rectus sheath and anchored there with interrupted sutures prolene 2/0 (onlay fashion). Haemostasis was secured and the suction drain was left in the subcutaneous space. Skin was closed with interrupted sutures. Intraoperative - post operative complications, wound complications, wound infection parameters evaluated.

\section{Results}

Previous abdominal operations were present in 1 patient. No patients had previous ventral hernia repairs. No difference existed in Operation times between all groups and mean operation time was (66 min 15+/- 35). In one patient minor bleeding occured from omental vessels and controlled intraoperatively. No other intraoperative complications occurred in any of the patients.

In the postoperative period, 1 patient developed wound haematoma and 1 patient developed seroma. 1 patient out of 9 developed wound infection, and 1 patient developed urinary retention postoperatively. No recurrence occured in 12 months follow-up.

\section{Discussion}

Umbilical hernias are the most common of all the abdominal hernias representing $6 \%$ of all abdominal hernias in adults [2,3]. 
The midline hernias abuting in the umbilicus superiorly or inferiorly are included in this group and are known as paraumbilical hernias [2,4]. They are more common in females [2,5]. Obesity, multiparity and prolonged labour are the predisposing factors to paraumbilical hernias $[2,6]$. The concept of repair of umbilical hernia in adults is not new, but the choice of appropriate surgical procedure is still a subject of debate $[2,7]$. The simple method of Mayo's repair has been commonly performed, but has the significant recurrence rate between $22-40 \%$. Nowadays, prosthetic mesh is frequently used to repair hernia defect [2,8]. More recently tension free hernioplasty has been performed for repair of umbilical hernias which has advantage over the Mayo's repair [2,9] The intersposition of prosthetic mesh not only reduces the tension but also avoids the re-approximation of avascular tissue and this explains the low recurrence rate [2].

Incarcerated hernias are frequently seen in the emergency ward. Usually, patients present with a painful swelling located on the ventral abdomen or groin. Some have signs of bowel obstruction, indicating incarceration or-at its worst-strangulation of the small or large bowel. The treatment of acute irreducible hernia consists of swift surgical exploration, with reduction of its contents and, if necessary, resection of ischaemic abdominal contents. Bowel resection produces a dilemma: the operation wound has become contaminated and is it, therefore, safe to use a mesh for correction? From the literature, it is known that primary suture repair in elective hernia repair increases the risk for recurrence-in many cases, leading to reoperation. This is the case in any type of abdominal wall hernia, whether ventral or inguinal [10-14].

The use of mesh in elective hernia repair has increased during the last two decades following large multicentre randomised controlled trials proving its superiority over primary suture to prevent recurrence [10]. However, this superiority has not been proven for acute irreducible hernias. Some smaller studies comparing mesh versus suture repair for this indication have been published, all denominating mesh repair to be safe and effective [10].

\section{Conclusion}

In this study all patients underwent tension-free mesh herniorrhaphy and the results demonstrated no elevated surgical complications and recurrence rates. Thus more studies with larger patients series needed.

\section{Bibliography}

1. Shankar DA., et al. "Factors associated with long-term outcomes of umbilical hernia repair". JAMA surgery 152.5 (2017): 461-466.

2. Tunio NA. "Hernioplasty: tension free mesh repair versus Mayos repair for umbilical hernias". Journal of Pakistan Medical Association 67.1 (2017): 24-26.
3. Stern EE. "Abdominal wall hernias". In: Stern EE. eds. Lang medical book of clinical thinking in Surgery, International Ed. Appleto: Lange, (1989): 393-395.

4. Abrahamson J Hernia. In: Schwartz. SI, Ellis H ed. Maingots abdominal Operation 9th ed. Norwalk Connecticut: Appleton and Lange, (1990): 256-266.

5. Ellis H. The abdomen In: Ellis H eds. clinical anatomy. 8thEd. Blackwell Scientific Publication, (1992): 57-62.

6. Sukeik M., et al. "Abdominal wall hernia during laproscopic Bypass: A Serious consideration". Obesity Surgery 17 (2007): 839-842.

7. Arroyo A., et al. "Mesh versus suture repair for umbilical hernias in adults. A prospective". British Journal of Surgery 88 (2001): 1321-1323.

8. Kelly R. "Radical cure of Para umbilical hernias". Annuals of Surgery 51 (1910): 694.

9. Du Bose M. "A new operation for Umilical hernia”. Surgery, Gynecology, and Obstetrics 21 (1915): 222-224.

10. Nieuwenhuizen Jeroen., et al. "The use of mesh in acute hernia: frequency and outcome in 99 cases". Hernia 15.3 (2011): 297-300.

11. Wysocki A., et al. "Is the Lichtenstein operation of strangulated groin hernia a safe procedure?" World Journal of Surgery 30.11 (2006): 2065-2070.

12. Wysocki A., et al. "Use of polypropylene prostheses for strangulated inguinal and incisional hernias". Hernia 5.2 (2001): 105-106.

13. Wysocki A., et al. "Lichtenstein repair for incarcerated groin hernias”. European Journal of Surgery 168 (2002): 452-454.

14. Shah RH., et al. "Laparoscopic repair of incarcerated ventral abdominal wall hernias". Hernia 12 (2008): 457-463.

\section{Volume 2 Issue 6 August 2019 \\ (C) All rights are reserved by Alp Yildiz., et al.}

